\title{
FLORA ARBUSTIVO-ARBÓREA DO FRAGMENTO DE FLORESTA ESTACIONAL SEMIDECIDUAL DO RIBEIRÃO CACHOEIRA, MUNICÍPIO DE CAMPINAS, SP1
}

\author{
Karin dos Santos ${ }^{2}$ \\ Luiza Sumiko Kinoshita ${ }^{2}$
}

Recebido em 17/07/1999. Aceito em 25/11/2002

\begin{abstract}
RESUMO - (Flora arbustivo-arbórea do fragmento de floresta estacional semidecidual do Ribeirão Cachoeira, município de Campinas, SP). Foi realizado inventário florístico das árvores e arbustos da mata Ribeirão Cachoeira (233,7ha, altitude $650 \mathrm{~m}$, coordenadas $46^{\circ} 55^{\prime} 58^{\prime \prime} \mathrm{W}, 22^{\circ} 50^{\prime} 13^{\prime}$ ' $\left.\mathrm{S}\right)$, o segundo maior e mais bem conservado fragmento de floresta estacional semidecidual do município de Campinas, SP. O solo característico é o Podzólico Vermelho Amarelo e o clima é Cwag' de Köppen. As coletas foram feitas durante o período de agosto/1996 a setembro/1997. Foram incluídos apenas indivíduos férteis com perímetro a altura do peito igual ou superior a $9 \mathrm{~cm}$. Foram encontradas 175 espécies de 119 gêneros e 49 famílias. As famílias mais ricas foram Myrtaceae (14 espécies), Rutaceae e Fabaceae (13), Caesalpiniaceae (11), Solanaceae (9) e Rubiaceae (8). Algumas espécies foram encontradas pela primeira vez na região: Tachigali multijuga Benth. e Schoepfia brasiliensis A.DC. A floração foi maior entre agosto e outubro. A frutificação foi maior nos meses de agosto a novembro. A maioria das espécies encontradas é zoocórica (58\%), as anemocóricas foram $23 \%$ e as autocóricas $19 \%$. Compararam-se as relações florísticas desta mata com outros 20 levantamentos do Estado. Os resultados obtidos indicaram a formação de dois grupos distintos. O mais homogêneo deles inclui as florestas do município de Campinas, mostrando que, possivelmente, são remanescentes de uma vegetação originalmente contínua.
\end{abstract}

Palavras-chave - florística, floresta semidecídua

ABSTRACT - (Floristic composition of the woody flora of the Ribeirão Cachoeira forest, Campinas, São Paulo State). An inventory of the woody flora (trees and shrubs), was carried out in the Ribeirão Cachoeria forest (233.7ha, 650 m high, 46 $55^{\prime} 58^{\prime \prime} \mathrm{W}, 22^{\circ} 50^{\prime} 13^{\prime \prime} \mathrm{S}$ ), the second largest and best conserved fragment of semideciduous tropical forest in the municipality of Campinas, São Paulo state, Southeastern Brazil. The soil is a red-yellow podsol and the climate is of Köppen's Cwag type. Collections were made from August/1996 to September/1997. Only fertile individuals with a perimeter at breast height of $9 \mathrm{~cm}$ or greater were included in the survey. One hyndred and seventhy five species were identified, belonging to 119 genera and 49 families. The most important families were Myrtaceae (14 species), Rutaceae and Fabaceae (13), Caesalpiniaceae (11), Solanaceae (9), and Rubiaceae (8). Some species were found for the first time in the region: Tachigali multijuga Benth. and Schoepfia brasiliensis A.DC. The flowering peak for most species was from August to October. Maximum fruit production was from August to November. Most species are zoochoric (58\%), but 23\% were anemochoric and 19\% autochoric. The floristic composition of this forest and another 20 forests from São Paulo state were compared. The results

1 Parte da Dissertação de Mestrado da primeira Autora; Bolsista FAPESP (ksbarbarella@ hotmail.com)

2 Departamento de Botânica, Instituto de Biologia, UNICAMP, C. Postal 6109, CEP 13083-970, Campinas, SP, Brasil (luizakin@unicamp.br) 
obtained indicate the existence of distinct groups of forests. The most homogeneus group contains forests from the municipality of Campinas with similarity of $40 \%$. This suggests that these forests are possibly fragments of a original continuous forest in the Campinas region.

Key words - floristics semideciduous forest

\section{Introdução}

No município de Campinas, há cerca de 197 fragmentos florestais que testemunham a vegetação original (Santin 1999). Dentro da Área de Proteção Ambiental (APA) de Souzas e Joaquim Egídio localiza-se o segundo maior e o mais bem preservado dentre estes fragmentos: a mata Ribeirão Cachoeira. A preservação e a recuperação deste e de outros remanescentes da APA são algumas das diretrizes da Secretaria de Planejamento e Meio Ambiente (SEPLAMA) da Prefeitura Municipal de Campinas (Miranda 1996).

As iniciativas em direção à preservação $\mathrm{e}$ recomposição de fragmentos requerem conhecimentos de florística, fitossociologia e também da ecologia de suas espécies componentes (Oliveira-Filho et al. 1994). Do ponto de vista florístico, alguns remanescentes de Campinas já foram estudados, entre eles citam-se Tamashiro et al. (1986), Matthes et al. (1988), Bernacci \& Leitão Filho (1996), Torres et al. (1994), Toniato et al. (1998), Spina (1997). No estudo de Salis et al. (1995) alguns destes levantamentos foram comparados e foi observada grande heterogeneidade entre eles.

A heterogeneidade entre fragmentos pode decorrer de variações de condições ambientais, das preferências ecológicas das espécies e também dos diversos graus de perturbação provocados pelo homem (Bertoni \& Martins 1987). Além disso, a fragmentação causa diminuição da diversidade e cada fragmento pode conter pequena parte da diversidade original (Denslow 1995). Assim, cada fragmento, com histórico e estado de preservação próprios, tornam-se únicos, o que aumenta sua importância para a conservação. Portanto, é funda- mental aumentar o conhecimento sobre as florestas remanescentes como a mata Ribeirão Cachoeira, pois o maior objetivo da conservação não é preservar um ideal de floresta intocada e sim a diversidade do ecossistema como um todo (Morellato \& Leitão Filho 1995).

Ainda, visando a conservação e manejo de remanescentes florestais, deve-se ressaltar a importância de uma abordagem ecológica. O estudo da fenologia fornece informações básicas da dinâmica das populações e dos aspectos interativos nas comunidades (Fournier 1976). A variação anual na produção de frutos e sementes influencia o recrutamento das populações e representa ainda importante componente no potencial de regeneração de floresta (Penhalber \& Mantovani 1997). O conhecimento de tais padrões é importante para a coleta de sementes e produção de mudas para reflorestamento, por exemplo.

Este trabalho objetivou estudar a composição florística da mata Ribeirão Cachoeira, comparar a listagem obtida às de outros remanescentes do Estado e fazer uma análise preliminar sobre os aspectos fenológicos e da dispersão.

\section{Material e métodos}

A mata Ribeirão Cachoeira tem área total de 233,7ha. Situa-se na porção nordeste do município ( $46^{\circ} 55^{\prime} 58^{\prime \prime} \mathrm{W}-22^{\circ} 50^{\prime} 13^{\prime}$ 'S), com um de seus vértices tangenciando as margens do rio Atibaia. As altitudes variam de 630 a $760 \mathrm{~m}$. O relevo é composto por morros, morrotes e morrotes paralelos com declividades de 12 a $30 \%$. O solo predominante é do tipo Podzólico Vermelho Amarelo. O clima, segundo a classificação de Köeppen (1948), é Cwag'temperado, moderadamente chuvoso, de inverno 
seco não rigoroso, com precipitação média anual de $1.409 \mathrm{~mm}$ (Mello et al. 1994). Ao longo de sua porção meridional corre o ribeirão Cachoeira, um riacho de substrato rochoso que forma numerosas corredeiras e pequenos saltos. A vegetação é densa, com altura de dossel variando entre 15 a $25 \mathrm{~m}$ e árvores emergentes de mais de $30 \mathrm{~m}$.

Para o estudo florístico, foi coletado material botânico fértil (em flor e/ou fruto) de indivíduos arbustivos ou arbóreos com PAP (perímetro a altura do peito) igual ou superior a $9 \mathrm{~cm}$. As coletas foram realizadas semanalmente de agosto/1996 a setembro/1997. O material coletado foi processado e identificado segundo técnicas usuais, no Laboratório de Taxonomia do Departamento de Botânica da Universidade Estadual de Campinas. As espécies foram incluídas em famílias de acordo com o sistema proposto por Cronquist (1981). A coleção principal de material herborizado foi incorporada ao herbário UEC e as duplicatas foram enviadas a outros herbários ou especialistas que ajudaram na identificação de alguns grupos.

As espécies foram separadas quanto à forma de crescimento, segundo terminologia proposta por Ramirez (1993). A forma de vida arvoreta foi considerada para os indivíduos férteis com altura inferior a $2 \mathrm{~m}$ e fuste único até $2 / 3$ de sua altura total.

$\mathrm{O}$ acompanhamento das fases fenológicas das espécies foi baseado, principalmente, nos dados de coleta. Para algumas espécies, foi feito acompanhamento de indivíduos marcados em campo. Entretanto, este acompanhamento não foi regular. Foram analisados os eventos de floração e frutificação e também as síndromes de dispersão apresentadas pelas espécies coletadas.

Foram realizadas comparações florísticas entre o conjunto de espécies encontradas no presente estudo e outros 20 levantamentos feitos no interior do Estado de São Paulo. A localização dos fragmentos comparados está representada na Fig. 1; seus autores, metodologia e algumas características dessas áreas, como tipo de solo e altitude encontram-se na Tab. 1.

Foi empregado o índice de similaridade de Jaccard (Pielou 1984). As relações de similaridade foram feitas através da análise de agrupamento UPGMA do programa FITOPAC (Shepherd 1995). Deve-se lembrar que em análises deste tipo, alguns fatores de ordem metodológica podem influenciar nos resultados. Como exemplo, pode-se citar o tamanho amostral e problemas taxonômicos, como os de identificação e sinonimização das espécies. Para minimizar tais efeitos na produção da matriz, as listagens florísticas foram sinonimizadas segundo revisões taxonômicas recentes e, somente os táxons identificados ao nível de espécie foram considerados. Além disso, foram incluídas apenas as espécies de hábito arbóreo, na tentativa de padronizar a amostra e reduzir interpretações errôneas que poderiam surgir ao se comparar listagens produzidas através de metodologias diferentes.

\section{Resultados e discussão}

Aspectos florísticos - na mata Ribeirão Cachoeira foram encontradas 175 espécies pertencentes a 119 gêneros e 49 famílias. Quanto à forma de vida, 150 espécies são arbóreas, totalizando $86 \%$ da amostra. Do restante, 12 espécies são arvoretas (7\%) e 13 são arbustos (7\%). Na Tab. 2 encontram-se as espécies amostradas no levantamento florístico em ordem alfabética de famílias e espécies, forma de vida e número do coletor.

As famílias mais ricas foram Myrtaceae (14 espécies), Rutaceae e Fabaceae (13), Caesalpiniaceae (11), Solanaceae (9), Rubiaceae (8), Moraceae, Meliaceae e Euphorbiaceae (7), Lauraceae e Mimosaceae (6). Estas onze famílias juntas somaram 101 espécies, constituindo $58 \%$ das espécies amostradas.

Considerando o estrato arbóreo, encontrou-se poucas famílias totalizando mais da metade das espécies observadas. Tais resultados 


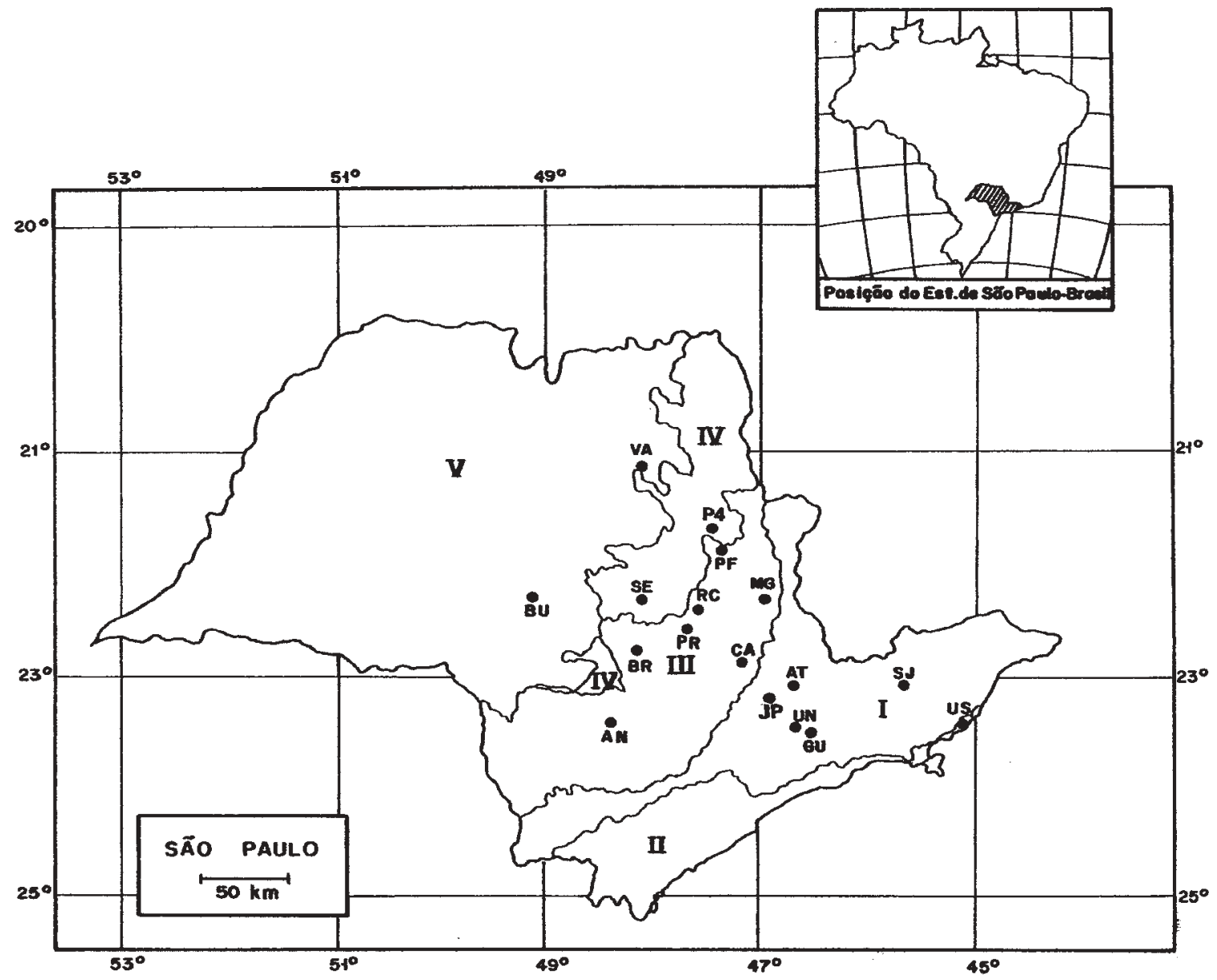

Figura 1. Localidades dos levantamentos florísticos incluídos no estudo de comparação florística. AN - Angatuba, Estação Ecológica de Angatuba (Torres 1989); AT - Atibaia, Parque Municipal da Grota Funda (Meira Neto et al. 1989); BR - Anhembi, Fazenda Barreiro Rico (Cesar \& Leitão Filho 1990); BU - Bauru, Reserva Estadual de Bauru (Cavassan et al. 1984); GU - Guarulhos, Floresta Remanescente do Aeroporto Internacional de Guarulhos (Gandolfi et al. 1995); PR - Piracicaba, Estação Ecológica de Ibicatu (Costa \& Mantovani 1995); JP - Jundiaí - Serra do Japi (Rodrigues et al. 1989); MG - Mogi-Guaçu, Mata da Figueira (Gibbs \& Leitão Filho 1978); PF - Porto Ferreira, Reserva Estadual de Porto Ferreira (Bertoni \& Martins 1987); P4 - Santa Rita do Passa Quatro, Parque Estadual de Vaçununga, Gleba Praxedes (Bertoni et al. 1988); RC - Rio Claro, Fazenda São José (Pagano \& Leitão Filho 1987); SE - Brotas, Fazenda Santa Elisa (Salis et al. 1994); SJ - São José dos Campos, Reserva Florestal Augusto Ruschi (Silva 1989); UB - Ubatuba, Sítio Experimental do Instituto Agronômico (Silva \& Leitão Filho 1982); UN - São Paulo, Cidade Universitária Armando de Salles Oliveira (Rossi 1994); VA - Matão, Mata da Virgínia (Rozza 1997); I - Planalto Atlântico; II - Província costeira, III - Depressão periférica; IV - Cuestas basálticas; V - Planalto Ocidental.

*CA - Florestas do município de Campinas: Bosque dos Jequitibás (Matthes et al. 1988), mata do Ribeirão Cachoeira (presente estudo), Reserva Municipal de Santa Genebra (Tamashiro et al. 1986), Fazenda São Vicente (Bernacci \& Leitão Filho 1996). 
Tabela 1. Características gerais dos levantamentos incluídos no estudo de comparação florística, os municípios, nome do remanescente, referência, metodologia utilizada, tipo de solo do fragmento, altitude (Alt., em metros) e número de espécies (n. esp.) de cada amostra. Li= solo litólico; LVA=Latossolo Vermelho Amarelo; AQ=solo Areia Quartzoza; $\mathrm{PVA}=$ solo Podzólico Vermelho Amarelo; LVE= Latossolo Vermelho Escuro LR= Latossolo Roxo.

\begin{tabular}{|c|c|c|c|c|c|}
\hline Levantamento & Referência & Método & Solo & Alt. & n. esp \\
\hline Angatuba (E.E. de Angatuba) & Torres 1989 & Florística & $\mathrm{Li}$ & 900 & 188 \\
\hline Anhembi (Faz. Barreiro Rico) & Cesar \& Leitão Filho 1990 & Florística & AQ & 500 & 83 \\
\hline Atibaia (P.M. da Grota Funda) & Meira Neto et al. 1989 & Florística & LVA & $900-1.400$ & 120 \\
\hline Bauru (R.E. de Bauru) & Cavassan et al. 1984 & Quadrantes & LR/LVE & 570 & 55 \\
\hline Brotas (Faz. Santa Elisa) & Salis et al. 1994 & Parcelas & LVE & 540 & 104 \\
\hline Campinas (B. dos Jequitibás) & Matthes et al. 1988 & Censo & LVA & 665 & 154 \\
\hline Campinas (Faz. São Vicente) & Bernacci \& Leitão Filho 1996 & Parcelas & PVA & $625-675$ & 143 \\
\hline Campinas (Mata do Ribeirão Cachoeira) & Presente estudo & Florí & PVA & $630-756$ & 174 \\
\hline Campinas (R.M. de Santa Genebra) & Tamashiro et. al. 1986 & Parcelas & - & 600 & 116 \\
\hline $\begin{array}{l}\text { Guarulhos (Aeroporto } \\
\text { Internacional de Guarulhos) }\end{array}$ & Gandolfi et al. 1995 & Parcelas & LVA & 740 & 140 \\
\hline Jundiaí (Serra do Japi) & Rodrigues et al. 1989 & Parcelas & LVA & 1020 & 115 \\
\hline Matão (Mata da Virgínia) & Rozza 1997 & $\begin{array}{l}\text { Parcelas/ } \\
\text { Floristica }\end{array}$ & PVA & 500 & 174 \\
\hline Mogi Guaçu (Mata da Figueira) & Gibbs \& Leitão Filho 1978 & Quadrantes & LVA & - & 46 \\
\hline Piracicaba (E.E. de Ibicatu) & Costa \& Mantovani 1995 & Parcelas & - & 740 & 102 \\
\hline Porto Ferreira (R.E. de Porto Ferreira) & Bertoni \& Martins 1987 & Quadrantes & - & $540-600$ & 81 \\
\hline Rio Claro (Faz. São José) & Pagano \& Leitão Filho 1987 & Quadrantes & LVA & 630 & 162 \\
\hline $\begin{array}{l}\text { Santa Rita do Passa Quatro } \\
\text { (P.E. de Vaçununga, Gleba Praxedes) }\end{array}$ & Bertoni et al. 1988 & Parcelas & LR/LVE & 640 & 56 \\
\hline $\begin{array}{l}\text { São José dos Campos } \\
\text { (R.F. Augusto Ruschi) }\end{array}$ & Silva 1989 & Parcelas & LVA & 840 & 174 \\
\hline $\begin{array}{l}\text { São Paulo (Cidade Universitária } \\
\text { Armando Salles de Oliveira) }\end{array}$ & Rossi 1994 & Florística & LVA & 750 & 116 \\
\hline Ubatuba (E.E. do Instituto Agronômico) & Silva \& Leitão Filho 1982 & Quadrantes & LVA & 105 & 101 \\
\hline
\end{tabular}

estão de acordo com a maioria dos estudos realizados em florestas estacionais semideciduais. Essas famílias, com exceção de Solanaceae, foram citadas por Leitão Filho (1982) como caracteristicamente abundantes nas matas do interior do Estado de São Paulo. Dentre elas, Myrtaceae tem sido mencionada como a mais rica em espécies em diversos levantamentos do interior do Estado: Matthes et al. (1988), Bernacci \& Leitão Filho (1996), Costa \& Mantovani (1995), Rozza (1997), Rodrigues et al. (1989), Gandolfi et al. (1995) e Salis et al. (1994). As demais famílias também figuram, com alguma variação de posição, entre as dez mais ricas nos levantamentos acima citados e nos de Cavassan et al. (1984), Pagano
\& Leitão Filho (1987) e Bertoni \& Martins (1987). Desse modo, a composição de famílias encontrada pode ser considerada a esperada para as florestas do Estado, em especial as florestas estacionais semideciduais. Nas formações florestais do interior do Estado de São Paulo, o conjunto de famílias mais ricas em espécies de arbóreas parece não variar muito de um fragmento para outro.

A quinta família mais rica no presente estudo, Solanaceae, foi citada entre as dez famílias mais ricas apenas por Gandolfi et al. (1995). Da mesma forma, o gênero Solanum, o mais rico em espécies no presente estudo, raramente figura entre os mais ricos em outros levantamentos. Tanto esta família como este 
Tabela 2. Relação das 49 famílias, 119 gêneros e 175 espécies encontrados na Mata Ribeirão Cachoeira - Campinas, $\mathrm{SP}$. Constam os números de coleta e forma de vida ( $\mathrm{Av}=$ arbórea; $\mathrm{Ab}=$ arbustiva; $\mathrm{At}=$ arvoreta).

ANACARDIACEAE

Astronium graveolens Jacq.

Tapirira obtusa (Benth.) J.D. Michell

ANNONACEAE

Annona cacans Warm.

Rollinia sylvatica (A. St.-Hil.) Mart.

Xylopia brasiliensis Spreng.

\section{APOCYNACEAE}

Aspidosperma cylindrocarpon Müll. Arg.

A. polyneuron Müll. Arg.

A. ramiflorum Müll. Arg.

Rauvolfia sellowii Müll. Arg.

ARALIACEAE

Dendropanax cuneatum Decne. \& Planch.

Didymopanax morototonii (Aubl.) Decne \& Planch.

ARECACEAE

Syagrus romanzoffiana (Cham.) Glassm.

ASTERACEAE

Gochnatia polymorpha (Less.) Cabrera

Vernonia discolor Less.

\section{BIGNONIACEAE}

Jacaranda micrantha Cham.

Zeyheria tuberculosa (Vell.) Bureau

BOMBACACEAE

Chorisia speciosa A. St.-Hil.

Eriotheca candolleana (K. Schum.) A. Robins

Pseudobombax grandiflorum (Cav.) A. Robins

\section{BORAGINACEAE}

Cordia ecalyculata Vell.

C. selowiana Cham.

C. trichotoma (Vell.) Arrabida ex Steud.

Patagonula americana $\mathrm{L}$.

Tournefortia rubicunda Salzm. ex A.DC.

\section{BURSERACEAE}

Protium heptaphyllum (Aubl.) Marchand

CAESALPINIACEAE

Bauhinia forficata Link

B. longifolia (Bongard) Steud.

Cassia ferruginea (Schrad) Schrad ex A. DC.

Copaifera langsdorffii Desf.

Holocalyx balansae Mich.

Hymenaea courbaril L.

Peltophorum dubium (Spreng.) Taub.

Schizolobium parahyba (Vell.) Blake

Senna multijuga (Rich.) H.S. Irwin \& Barneby

S. spectabilis (DC.) H.S. Irwin \& Barneby

Tachigali multijuga Benth.

CARICACEAE

Jacaratia spinosa (Aubl.) A.DC.
$\mathrm{Av}$

$\mathrm{Av}$

$\mathrm{Av}$

$\mathrm{Av}$

$\mathrm{Av}$

$\mathrm{Av}$

$\mathrm{Av}$

$\mathrm{Av}$

Av

$\mathrm{Av}$

$\mathrm{Av}$

$\mathrm{Av}$

Av

Av

$\mathrm{Av}$

$\mathrm{Av}$

Av

$\mathrm{Av}$

$\mathrm{Av}$

Av

Av

Av

Av

$\mathrm{Ab}$

Av

Av

Av

Av

Av

Av

Av

Av

Av

Av

Av

$\mathrm{Av}$

Av
26; 103

289

293

$95 ; 129 ; 193$

226

59

$24 ; 75$

291

$138 ; 211 ; 248$

269

282

88; 253

$51 ; 114 ; 275 ; 283$

288

284

201

270

264; 280

160; 202

$210 ; 221 ; 229$

277

148; $149 ; 156$

$98 ; 170$

157

$76 ; 162$

$172 ; 220$

163

191; 247

89

3; 146

192

281

180; 204

194

225

90 
Tabela 2 (continuação)

\section{CECROPIACEAE}

Cecropia glaziovii Snethl.

C. hololeuca Miq.

CELASTRACEAE

Maytenus aquifolium Mart.

M. robusta Reiss.

CHRYSOBALANACEAE

Hirtella hebeclada Moric. ex A. DC.

ERYTHROXYLACEAE

Erythroxylum deciduum A. St.-Hil.

\section{EUPHORBIACEAE}

Actinostemon communis (Müll. Arg.) Pax

A. concolor (Spreng.) Müll. Arg.

Alchornea glandulosa Poepp. \& Endl.

A. triplinervea (Spreng.) Müll. Arg.

Croton floribundus Spreng.

C. priscus Croizat

Savia dictyocarpa Müll. Arg.

FABACEAE

Centrolobium tomentosum Benth.

Erythrina falcata Benth.

Lonchocarpus campestris Mart ex Benth.

L. guilleminianus (Tul.) Malme

L. muehlbergianus Hassl.

Luetzelburgia auriculata (Fr. All.) Ducke

Machaerium hirtum Raddi

M. nyctitans (Vell.) Benth.

M. scleroxylon Tul.

M. villosum Vogel

Myroxylon peruiferum L.f.

Ormosia arborea (Vell.) Harms

Sweetia fruticosa Spreng.

FLACOURTIACEAE

Casearia decandra Jacq.

C. gossypiosperma Briquet

C. sylvestris Swartz

Prockia crucis $\mathrm{P}$. Browne ex L.

ICACINACEAE

Citronella megaphyla (Miers) Howard

LACISTEMATACEAE

Lacistema hasslerianum Chodat

LAURACEAE

Aniba firmula (Ness \& Martius ex Ness) Mez

Cryptocarya aschersoniana Mez

Nectandra megapotamica (Spreng.) Mez

Ocotea beulahiae Baitello

O. indecora (Schott) Mez

O. puberula (Rich.) Nees

LECYTHIDACEAE

Cariniana estrellensis (Raddi) Kunth.
267

$\mathrm{Av}$

$\mathrm{Av}$

At

Av

268

147

258

Av

$121 ; 140$

Av

Av

Av

$\mathrm{Av}$

$\mathrm{Av}$

$\mathrm{Av}$

$\mathrm{Av}$

Av

Av

Av

Av

Av

Av

Av

Av

Av

Av

Av

Av

Av

Av

Av

Av

Av

At

Av

$\mathrm{Ab}$

Av

Av

Av

Av

Av

Av

Av
$184 ; 261$

$91 ; 123$

\section{4; 175}

$36 ; 116 ; 285$

$23 ; 37 ; 286$

132; 265

287

$84 ; 107 ; 164$

$85 ; 165$

227

215

263

154

$185 ; 240$

$155 ; 177$

292

218

$33 ; 92 ; 241$

206

34; 178

179; 254

279

235

105; 137

$55 ; 196$

$15 ; 28 ; 29$

141

133

86; 173

47; 48; 126

$21 ; 78$

212

$167 ; 195 ; 274$

$7 ; 152$ 
Tabela 2 (continuação)

\section{LECYTHIDACEAE}

C. legalis (Mart.) Kunth.

LOGANIACEAE

Strychnos brasiliensis (Spreng.) Mart.

MALVACEAE

Abutilon longifolium K. Schum.

A. peltatum K. Schum.

Bastardiopsis densiflora (Hook. \& Arn.) Hass.

MELASTOMATACEAE

Miconia discolor A. DC.

M. petropolitana Cogn.

M. pusiliflora Triana

Ossaea sanguinea Cogn.

MELIACEAE

Cabralea canjerana (Vell.) Mart.

Cedrela fissilis Vell.

Guarea macrophylla Vahl

Trichilia catigua A. Juss.

T. claussenii C. DC.

T. elegans A. Juss.

T. pallida Swartz

MIMOSACEAE

Acacia polyphylla DC.

A. paniculata Willd.

Calliandra foliolosa Benth.

Inga luschnatiana Benth.

I. marginata Willd.

Piptadenia gonoacantha (Mart.) J.F. Macbr.

MONIMIACEAE

Mollinedia elegans Tul.

M. widgrenii A. DC.

MORACEAE

Brosimum gaudichaudii Trec.

Ficus arpazuza Casaretto

F. glabra Vell.

F. guaranitica Chodat ex Chodat \& Vischer

F. insipida Willd.

F. obtusifolia H.B.K.

Maclura tinctoria D. Don ex Steud.

MYRSINACEAE

Rapanea umbellata (Mart.) Mez

MYRTACEAE

Calycorectes acutatus (Miq.) Toledo

Calyptranthes clusiifolia (Miq.) O. Berg

Campomanesia guazumaefolia (Cambess.) O. Berg

C. neriiflora (O. Berg) Nied.

Eugenia burkartiana (D. Legrand) D. Legrand

E. excelsa O. Berg

E. glazioviiana Kiaersk.

E. leptoclada O. Berg
Av

$\mathrm{Ab}$

$\mathrm{Ab}$

$\mathrm{Ab}$

Av

Av

Av

Av

$\mathrm{Ab}$

Av

Av

Av

Av

Av

At

Av

Av

Av

Av

Av

Av

Av

At

Av

Av

Av

Av

Av

Av

Av

Av

Av

Av

Av

Av

Av

Av

Av

Av

Av

\section{8}

130

4; 237

$12 ; 238$

$13 ; 60 ; 272$

$70 ; 250$

$242 b$

19; $25 ; 46 ; 242$

127

$14 ; 61 ; 62$

$64 ; 65 ; 82 ; 228 ; 276$

$17 ; 69 ; 111 ; 145 ; 260$

$99 ; 131 ; 214 ; 222$

$11 ; 22$

251

$50 ; 100 ; 219$

188

$27 ; 200$

$38 ; 63 ; 134$

$32 ; 52$

153

2; 168

$20 ; 232$

$58 ; 113 ; 187$

73

231

278

31

109; 230; 249

176

93; 94

262

$104 ; 118 ; 135$

266

136

151

181

42

120

67 
Tabela 2 (continuação)

\section{MYRTACEAE}

E. ligustrina (Sw.) Willd.

Av

Gomidesia affinis (Cambess.) D. Legrand

Myrcia richardiana $\mathrm{O}$. Berg

M. rostrata DC.

Myrciaria floribunda (West ex Willd.) O. Berg

Psidium guajava $\mathrm{L}$.

NYCTAGINACEAE

Guapira opposita (Vell.) Reitz

OLACACEAE

Schoepfia brasiliensis A. DC.

PHYTOLACCACEAE

Gallesia integrifolia (Spreng.) Harms

Seguieria langsdorffii Moq.

PROTEACEAE

Roupala brasiliensis Klotzsch

RHAMNACEAE

Colubrina glandulosa Perk.

Rhamnidium elaeocarpus Reissek

\section{ROSACEAE}

Prunus myrtifolia (L.) Urb.

\section{RUBIACEAE}

Alseis floribunda Schott.

Ixora gardneriana Benth.

I. venulosa Benth.

Psychotria sessilis (Vell.) Müll. Arg.

Randia armata (Sw.) DC.

Guettarda uruguensis Cham. \& Schlecht.

Rudgea apoda Müll. Arg.

R. jasminoides (Cham.) Müll. Arg.

\section{RUTACEAE}

Almeidea coerulea St.Hil. ex G. Don.

Balfourodendron riedelianum (Engl.) Engl.

Conchocarpus pentandrus (A. St.Hil.) Kallumki \& Pirani

Esenbeckia grandiflora Mart.

E. leiocarpa Engl.

Galipea multiflora Shultz

Metrodorea nigra A. St.-Hil.

M. stipularis Mart.

Zanthoxylum hyemale A. St.-Hil

Z. minutiflorum Tul.

Z. monogynum A. St.-Hil.

Z. petiolare A. St.-Hil. \& Tul.

Z. rhoifolium Lam.

\section{SAPINDACEAE}

Cupania vernalis Camb.

Matayba elaeagnoides Radlk.
$\mathrm{Av}$

Av

Av

Av

Av

Av

Av

Av

Av

Av

Av

Av

Av

Av

Av

$\mathrm{Ab}$

Av

$\mathrm{Ab}$

At

At

Av

Av

Av

$\mathrm{Ab}$

At

Av

At

Av

Av

Av

Av

Av

Av

Av

Av

Av
68; 139; 257

205; 216

161

124

125

169

$43 ; 56 ; 110$

243

252

96

1

66; 106

158

271

81; 102; 208

79

182

9; 122

142

53; 236

203

$80 ; 183 ; 190$

5

150

39

41; 189

256

16; 198

$10 ; 18 ; 45$

57

$35 ; 101 ; 255$

144; 159

115

290

199
$40 ; 87$

77; 112; 166; 186 
Tabela 2 (continuação)

Família/Espécie
Forma de Vida n. do coletor

(série de K. Santos)

\begin{tabular}{|c|c|c|}
\hline \multicolumn{3}{|l|}{ SAPOTACEAE } \\
\hline Chrysophyllum gonocarpum (Mart. \& Eichl.) Engl. & Av & $83 ; 119 ; 246$ \\
\hline C. marginatum (Hook. \& Arn.) Radlk. & At & 197 \\
\hline \multicolumn{3}{|l|}{ SIMAROUBACEAE } \\
\hline Picramnia ramiflora Planchon & At & 128 \\
\hline \multicolumn{3}{|l|}{ SOLANACEAE } \\
\hline Cestrum intermedium Sendt. & $\mathrm{Ab}$ & 108 \\
\hline Brugmansia suaveolens Ber. \& Presl & $\mathrm{Ab}$ & 239 \\
\hline Solanum argenteum Dunal & At & 71 \\
\hline S. granuloso-leprosum Dunal & Av & $97 ; 233$ \\
\hline S. lycocarpum A.St.-Hil. & Av & $223 ; 244$ \\
\hline S. pseudoquina A.St.-Hil & Av & $72 ; 213$ \\
\hline S. robustum Wendl. & $\mathrm{Ab}$ & 224 \\
\hline S. sanctae-catharinae Dunal & At & 143 \\
\hline Solanum sp. & Av & 234 \\
\hline \multicolumn{3}{|l|}{ STYRACACEAE } \\
\hline Styrax glabratum Spreng. & $\mathrm{Av}$ & 259 \\
\hline \multicolumn{3}{|l|}{ TILIACEAE } \\
\hline Heliocarpus americanus L. & Av & 273 \\
\hline Luehea divaricata Mart. & Av & $44 ; 217$ \\
\hline \multicolumn{3}{|l|}{ ULMACEAE } \\
\hline Celtis iguanae (Jacq.) Sargent & $\mathrm{Ab}$ & 209 \\
\hline Trema micrantha (L.) Blume & $\mathrm{Av}$ & 49 \\
\hline \multicolumn{3}{|l|}{ VERBENACEAE } \\
\hline Aegiphila sellowiana Cham. & Av & 117 \\
\hline A. lhotzkyana Cham. & Av & $171 ; 245$ \\
\hline Aloysia virgata (Ruiz \& Pav.) A.Juss. & Av & 54 \\
\hline Citharexylum myrianthum Cham. & Av & 174 \\
\hline \multicolumn{3}{|l|}{ VOCHYSIACEAE } \\
\hline Vochysia tucanorum Mart. & $\mathrm{Av}$ & 207 \\
\hline
\end{tabular}

gênero apresentam muitas espécies de pequeno porte, freqüentemente ocorrendo em bordas de mata e áreas mais abertas. Em Ribeirão Cachoeira, quatro das sete espécies do gênero Solanum são arvoretas ou arbustos e foram coletados em áreas de borda ou clareiras. Talvez por isso não figurem entre os mais ricos nos demais trabalhos citados. A metodologia pode ser o principal fator determinante deste fato, pois tanto os métodos de levantamento (parcelas e transectos no interior da mata) quanto os critérios de inclusão considerados (diâmetros e/ou perímetros mínimos do tronco) variam entre os trabalhos consultados, influenciando as listagens obtidas. Naqueles em que a família Solanaceae apresenta elevado número de espécies, os autores se referem à forma de vida herbáceoarbustiva, como é o caso de Bernacci \& Leitão Filho (1996) e Meira Neto et al. (1989).

Os gêneros com maior número de espécies em Ribeirão Cachoeira foram: Solanum, com sete espécies; Ficus, Eugenia e Zanthoxylum, com cinco espécies; Trichilia e Machaerium com quatro espécies cada. Seis gêneros contribuiram com três espécies cada: Ocotea, Cordia, Aspidosperma, Lonchocarpus, Casearia e Miconia; 21 gêneros contribuíram com duas espécies e 87 com apenas uma espécie. 
Dentre as arbóreas, Ocotea foi citado como o gênero mais rico em espécies por Matthes et al. (1988) e Gibbs \& Leitão Filho (1978). Por outro lado, Bernacci \& Leitão Filho (1996) e Bertoni \& Martins (1987) encontraram Eugenia como o gênero mais rico. Ainda, Cavassan et al. (1984) citaram Cordia, Trichilia e Casearia como os gêneros com maior número de espécies e Martins (1991) mencionou apenas Trichilia. Todos estes gêneros foram bem representados em Ribeirão Cachoeira. Constatou-se, assim, que esses gêneros estão sempre bem representados entre as espécies arbóreas, figurando entre os dez primeiros em número de espécies em diversos levantamentos.

Da mesma forma, a grande maioria das espécies aqui encontradas, freqüentemente aparece em levantamentos florísticos de mata estacional semidecidual. Algumas delas como: Acacia polyphylla, Aspidosperma polyneuron, Astronium graveolens, Annona cacans, Casearia gossypiosperma, Cariniana estrellensis, Cedrela fissilis, Centrolobium tomentosum, Chrysophyllum gonocarpum, Copaifera langsdorffii e Trichilia catigua foram mencionadas por Salis et al. (1995) como espécies representativas desse tipo de mata.

Outras espécies deste levantamento Prunus myrtifolia, Rapanea umbellata, Myrcia rostrata e Cordia sellowiana - foram consideradas indicadoras de florestas de altitude por Meira Neto et al. (1989). Enquanto Alseis floribunda, Aniba firmula, Cryptocaria aschersoniana e Schoepfia brasiliensis são, segundo Leitão Filho (1993), espécies que têm como área primária de ocorrência a floresta tropical atlântica de encosta da Serra do Mar.

Tais generalizações, apesar de tentarem fornecer visão sucinta da vegetação, devem ser encaradas com cautela, pois espécies típicas de determinadas formações vegetais podem coexistir com outras mais freqüentes em outros tipos de formação. Assim, as espécies podem ser mais freqüentes e abundantes em uma formação vegetal e podem ser raras ou ausentes em outras (Leitão Filho 1993). Deve-se lembrar também que essas florestas ocupavam originalmente grande parte do sul, sudeste e centro-oeste do Brasil, eram razoavelmente contínuas e entremeadas por outros tipos vegetacionais, como cerrados, matas de brejo, matas de galeria, onde as espécies peculiares de cada formação se misturavam (Bertoni \& Martins 1987; Salis et al. 1994; Grombone et al. 1990). Com a fragmentação, restaram manchas pontuais de vegetação muito heterogêneas entre si. Isto contribui para que algumas espécies estejam presentes em algumas localidades e ausentes em outras ou ocorram raramente nos remanescentes estudados.

Assim, a importância de estudos como este da mata Ribeirão Cachoeira está muito mais em apontar onde as espécies estão ocorrendo, do que em definir limites para as diferentes formações vegetais. Dentro desse contexto, vale salientar que a ocorrência de Tachigali multijuga (Caesalpiniaceae) e Schoepfia brasiliensis (Olacaceae) estão sendo citadas pela primeira vez para o município de Campinas, e que a ocorrência destas espécies é rara também para o Estado de São Paulo. Ainda, Aniba firmula e Cryptocaria aschersoniana (Lauraceae), Aegiphila lhotzkyana (Verbenaceae) e Alseis floribunda (Rubiaceae) raramente são citadas em outros estudos das matas do interior do Estado de São Paulo. Tais observações acentuam a importância da preservação da mata Ribeirão Cachoeira, especialmente se considerarmos seu melhor estado de conservação em relação aos demais remanescentes da cidade de Campinas, e sua proximidade geográfica a um importante centro de pesquisas como a UNICAMP.

Aspectos fenológicos - durante o ano de observação, a maioria das espécies (66\%) floresceu entre o final da estação seca (julhosetembro) e o início do estação úmida (outubronovembro) (Fig. 2). Tal padrão foi também observado por Jackson (1978), Morellato et al. (1989), Rossi (1994), Baider (1994), Stranghetti 
(1996) e Penhalber \& Mantovani (1997). Para Jansen (1967), este período favorece a polinização, pois a escassez de chuvas permite maior concentração de néctar e a permanência das flores nos ramos por mais tempo. Além disso, a queda das folhas nesta época permite melhor vizualização das flores e melhor deslocamento dos polinizadores. Frankie et al. (1974) atribuem o termo "floração sazonal" ("seasonal flowering") quando a maioria das espécies floresce num único período, fenômeno típico de ambientes tropicais com estações bem definidas.

A frutificação pareceu ocorrer mais uniformemente do que a floração durante o ano de observação (Fig. 2). Tal uniformidade pode ser devida ao fato de que a maioria das espécies em Ribeirão Cachoeira é zoocórica. Este padrão de dispersão é exibido por 102 espécies ou 58\% da amostra. As espécies zoocóricas geralmente apresentam padrão contínuo de frutificação, produzindo frutos durante todo o ano, o que caracteriza frutificação seqüencial (Morellato \& Leitão Filho 1992).

A maior proporção de espécies com síndrome de dispersão zoocórica é comum nas florestas tropicais (Morellato \& Leitão Filho 1992; Penhalber \& Mantovani 1997; Rossi 1994). As demais síndromes ocorrem em menor proporção, assim como o observado em Ribeirão Cachoeira, onde a anemocoria foi o padrão de dispersão de apenas $23 \%$ da amostra (40

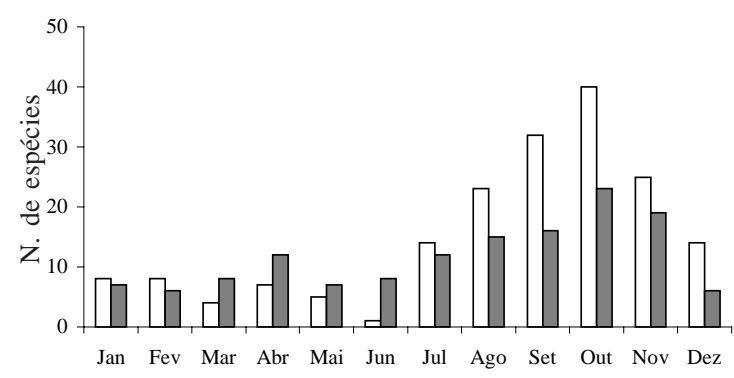

Figura 2. Número de espécies lenhosas em floração e frutificação ao longo do ano (agosto/1996 a setembro/1997), na mata Ribeirão Cachoeira, Campinas, SP. $\square$ Floração; $\square$ Frutificação. espécies). As autocóricas foram 33 espécies, correspondendo a $19 \%$ da amostra (Fig. 3).

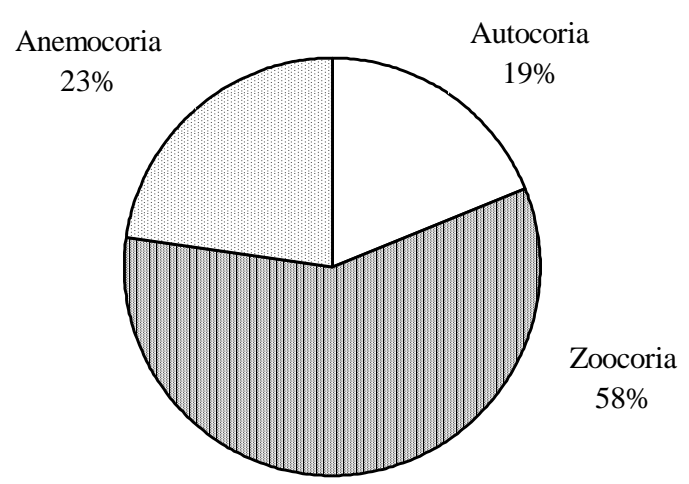

Figura 3. Síndromes de dispersão apresentadas pelas espécies arbustivas e arbóreas da mata Ribeirão Cachoeira, Campinas, SP.

No período de agosto a novembro (final da estação seca e início da estação úmida) houve ligeiro aumento do número de coletas e/ou observações de espécies com frutos. Tal aumento foi devido às espécies anemocóricas, que dispersam seus frutos no final da estação seca, e às espécies que aproveitam o início da estação úmida para produção e/ou dispersão de frutos. Nesta época, as condições são mais favoráveis à germinação e estabelecimento das plântulas, devido à maior disponibilidade de água e luz, antes da próxima estiagem (Morellato \& Leitão Filho 1992). Outros autores também observaram aumento na frutificação em período semelhante (Penhalber \& Mantovani 1997; Rossi 1994). Este tipo de estratégia foi descrito por Fournier \& Salas (1966).

Apesar de não ter havido esforço amostral específico para o comportamento fenológico na mata Ribeirão Cachoeira, foi observado que os eventos fenológicos, principalmente a floração, parecem ser sazonais, como o encontrado por outros autores em florestas submetidas a condições climáticas sazonais. É aconselhável, entretanto, aumentar o tempo de observação, para a obtenção de informações mais seguras. 
Sugere-se que os dados de floração e frutificação, principalmente aqueles referentes às espécies menos freqüentes nas matas do Estado, são muito importantes para o estabelecimento de ações de manejo, preservação e recomposição. Além disso, a grande proporção de espécies zoocóricas encontrada ressalta que é fundamental a manutenção e a conservação da mata, pois é suporte para sua fauna e também, provavelmente, para a fauna da região, se considerarmos o contexto da paisagem.

Comparação florística - remanescentes de mata podem ser considerados semelhantes sempre que apresentarem pelo menos $25 \%$ de concordâncias (Müller-Dumbois \& Ellenberg 1974). Este índice, apesar de subjetivo, tem auxiliado os pesquisadores no estudo dos remanescentes de mata e no entendimento de suas semelhanças e particularidades. De modo geral, foram observados baixos índices de similaridade entre os fragmentos comparados.

Por intermédio da análise do dendrograma de similaridade obtido (Fig. 4), pode-se perceber a formação de dois grupos bem conspícuos, que exibiram apenas $17 \%$ de similaridade. O primeiro é formado pelas florestas da Bacia de São Paulo: São Paulo (UN), Guarulhos (GU), São José dos Campos (SJ) e pelas matas consideradas de altitude: Angatuba (AN), Atibaia (AT) e Serra do Japi (JP). O segundo é formado pelas matas de planalto do interior do Estado como as matas de Campinas (RB, SV, SG, BJ), Rio Claro (RC), Matão (VA) e Bauru (BU). Incluem-se neste último, matas de galeria como Fazenda Santa Elisa em Brotas (SE), Mata da Figueira em MogiGuaçu (MG) e a Reserva de Porto Ferreira (PF). A formação destes dois grandes grupos foi observada por outros autores em estudos semelhantes.

Para Salis et al. (1995), este resultado pode ser explicado pela influência da altitude. Torres et al. (1997) definiram tais agrupamentos florísticos como "mesophyllous high altitudinal" e "mesophyllous low-altitudinal" em alusão às maiores altitudes das matas do primeiro grupo e às altitudes menores no segundo grupo (Tab. 1). Além disso, estes autores salientam que os solos ocupados pelas matas de altitude são quase sempre distróficos e sofrem influência maior de geadas no inverno.

No grupo das florestas de planalto pode-se perceber a formação de agrupamentos menores (Fig. 4). O primeiro subgrupo é formado pelas matas da Reserva de Porto Ferreira (PF) e Mata da Figueira em Mogi-Guaçu (MG), ambas florestas de galeria, que sofrem grande influência da umidade do solo. O segundo subgrupo é composto pela Reserva Estadual de Bauru (BU) e a Reserva Estadual de Vaçununga, Gleba Praxedes, em Santa Rita do Passa Quatro (P4); este agrupamento também foi obtido por Salis et al. (1995). A similaridade apresentada pode ser atribuída ao mesmo tipo de solo ocupado por estes remanescentes (Tab. 1). O terceiro subgrupo é constituído pela Estação Ecológica de Ibicatu (PR), Fazenda Santa Elisa (SE), Mata

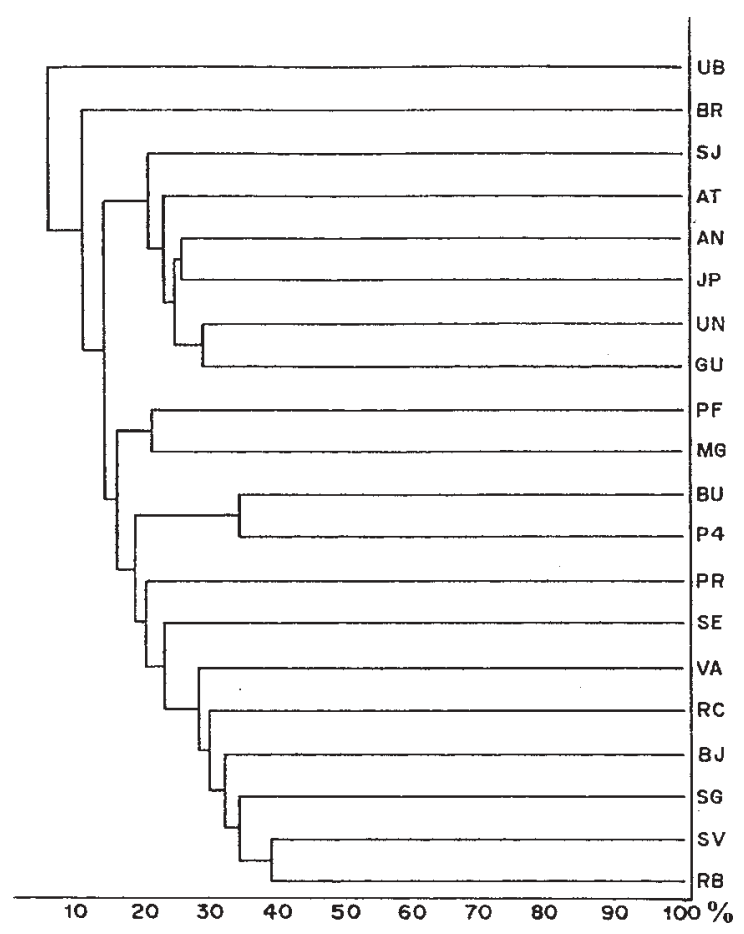

Figura 4. Dendrograma de similaridade obtido através do índice de Jaccard entre 20 levantamentos florísticos do Estado de São Paulo (vide legenda da Fig. 1). 
da Vírginia (VA), Fazenda São José (RC) e as florestas do município de Campinas. No interior do Estado, é comum as matas de galeria apresentarem grande número de espécies de mata estacional semidecidual, o que explicaria a inclusão de matas de galeria como MG, SE e PF junto às demais florestas (Salis et al. 1994; Costa \& Mantovani 1995). Tanto as semelhanças como as diferenças dentro deste grande grupo poderiam ser explicadas por parâmetros do solo como fertilidade, textura e categoria taxonômica, além de graus de perturbação e estádio sucessional (Torres et al. 1997).

Alguns levantamentos aparecem isolados dos demais grupos, como Anhembi (BR) e Ubatuba (UB) (Fig. 4). A baixa similaridade entre o remanescente de Anhembi e os demais pode ser devida ao fato de que esta área tem grande influência de vegetação de Cerrado e é composta por diversos tipos de ambientes e matas. No caso de Ubatuba (UB), explica-se seu isolamento por se tratar de outra formação vegetal, floresta tropical atlântica de encosta da Serra do Mar, com altitude e pluviosidade bastante diferenciadas (Tab. 1).

As matas do município de Campinas apresentaram similaridade em torno de 30 a $40 \%$, formando agrupamento bastante conspícuo (Fig. 4). A maior semelhança, $40 \%$, foi encontrada entre a mata Ribeirão Cachoeira (RB) e a mata Fazenda São Vicente (SV). Estes dois remanescentes exibem histórico de perturbação e ocupação semelhantes e menos intensas que o Bosque do Jequitibás e a Reserva da Santa Genebra. Estes dois últimos exibiram menor similaridade tanto entre si quanto em relação a Ribeirão Cachoeira e Fazenda São Vicente. O Bosque dos Jequitibás ocupa área central da cidade e a Reserva da Santa Genebra tem histórico de perturbação bastante intenso. Os quatro remanescentes de Campinas apresentaram 24 espécies em comum (Tab. 3). As maiores concordâncias ocorreram entre as matas de Ribeirão Cachoeira e Fazenda São Vicente, com 80 espécies em comum. Segue-se
Ribeirão Cachoeira e Bosque dos Jequitibás, com 73 espécies em comum e Ribeirão Cachoeira e Reserva Santa Genebra, com 68 espécies.

Os resultados aqui obtidos, com alguma variação, estão de acordo com a maioria das comparações realizadas por outros autores no Estado de São Paulo como, Salis et al. (1995), Costa \& Mantovani (1995) e Torres et al. (1997). Assim, fatores abióticos como clima, topografia e tipo de solo podem estar influenciando a composição florística dos remanescentes de mata e, conseqüentemente, a similaridade entre eles. Deste modo, a maior similaridade florística observada entre os remanescentes do município de Campinas poderia ser explicada não apenas por sua

Tabela 3. Espécies arbóreas comuns aos quatro remanescentes do município de Campinas: Ribeirão Cachoeira (presente estudo); Reserva de Santa Genebra (Tamashiro et al. 1986); Bosque dos Jequitibás (Matthes et al. 1988) e Fazenda São Vicente (Bernacci \& Leitão Filho 1996).

\begin{tabular}{|c|c|}
\hline Espécie & Família \\
\hline Aegiphila sellowiana & VERBENACEAE \\
\hline Aspidosperma polyneuron & APOCYNACEAE \\
\hline Aspidosperma ramiflorum & APOCYNACEAE \\
\hline Astronium graveolens & ANACARDIACEAE \\
\hline Bauhinia forficata & CAESALPINIACEAE \\
\hline Cariniana legalis & LECYTHIDACEAE \\
\hline Casearia gossypiosperma & FLACOURTIACEAE \\
\hline Casearia sylvestris & FLACOURTIACEAE \\
\hline Cedrela fissilis & MELIACEAE \\
\hline Chorisia speciosa & BOMBACACEAE \\
\hline Croton priscus & EUPHORBIACEAE \\
\hline Eriotheca candolleana & BOMBACACEAE \\
\hline Esenbeckia leiocarpa & RUTACEAE \\
\hline Hymenaea courbaril & CAESALPINIACEAE \\
\hline Jacaranda micrantha & BIGNONIACEAE \\
\hline Machaerium nictitans & FABACEAE \\
\hline Metrodorea nigra & RUTACEAE \\
\hline Miconia hymenonervia & MELASTOMATACEAE \\
\hline Myroxylon peruiferum & FABACEAE \\
\hline Ormosia arborea & FABACEAE \\
\hline Securinega guaraiuva & EUPHORBIACEAE \\
\hline Trema micrantha & ULMACEAE \\
\hline Trichilia pallida & MELIACEAE \\
\hline Xylopia brasiliensis & ANNONACEAE \\
\hline
\end{tabular}


proximidade geográfica, mas também pela uniformidade climática e de cotas de altitude (entre 550 e $750 \mathrm{~m}$ ) existente entre eles. Os resultados obtidos também podem estar indicando que, provavelmente, no passado, a vegetação do município de Campinas era constituída por cobertura vegetal contínua e que hoje apresenta-se fragmentada, com remanescentes florestais entremeados por áreas urbanas e rurais. Assim, o processo de fragmentação pode estar influenciando para que os remanescentes se tornem cada vez mais distintos entre si.

\section{Agradecimentos}

À FAPESP e FAEP - Unicamp, pelo apoio financeiro; Maria Lúcia Kawasaki, Renato Goldenberg, Jorge Tamashiro, Pedro Carauta, José Rubens Pirani, Reinaldo Monteiro, Charlote Taylor e João B. Baitello, pelo auxílio nas identificações dos grupos mais difíceis. A Antônio Geremias, pela ajuda nos trabalhos de campo.

\section{Referências bibliográficas}

Baider, C. 1994. O banco de sementes e de plântulas na sucessão da Mata Atlântica, São Paulo. Dissertação de Mestrado. Universidade Estadual de São Paulo, São Paulo.

Bernacci, L. C. \& Leitão Filho, H. F. 1996. Flora fanerogâmica da floresta da Fazenda São Vicente, Campinas, SP. Revista Brasileira de Botânica 19(2): 149-164.

Bertoni, J. E. A. \& Martins, F. R. 1987. Composição florística de uma floresta ripária na Reserva Estadual de Porto Ferreira, SP. Acta Botanica Brasilica 1(1): 17-26.

Bertoni, J. E. A.; Martins, F. R.; Moraes, J. L. \& Shepherd, G. J. 1988. Composição florística e estrutura fitossociológica do Parque Estadual de Vaçununga, Santa Rita do Passa Quatro - Gleba Praxedes. Boletim Técnico do Instituto Florestal de São Paulo 42: 149-170.

Cavassan, O.; Cesar, O. \& Martins, F. R. 1984. Fitossociologia da vegetação arbórea da reserva estadual de Bauru, Estado de São Paulo. Revista Brasileira de Botânica 7(2): 91-106.
Cesar, O. \& Leitão Filho, H. F. 1990. Estudo florístico quantitativo de mata mesófila semidecídua na fazenda Barreiro Rico, município de Anhembi, SP. Revista Brasileira de Botânica 50(1): 133-147.

Costa, L. G. S. \& Mantovani, W. 1995. Flora arbustivoarbórea de trecho de mata mesófila semidecídua, no sítio ecológico de Ibicatu, Piracicaba (SP). Hoehnea 22(1/2): 47-59.

Cronquist, A. 1981. An integrated system of classification of flowering plants. Columbia University Press, New York.

Denslow, J. S. 1995. Disturbance and diversity in tropical rain forests: The density effect. Ecological Aplications 5: 962-968

Fournier, L. A. 1976. Observaciones fenológicas en el bosque húmedo de pré-montano de San Pedro de Monte de Oco, Costa Rica. Turrialba 26: 54-59.

Fournier, L. A. \& Salas, S. 1966. Algunas observaciones sobre la dinámica de la floración en el bosque húmedo de Villa Colon. Revista de Biologia Tropical 14: 75-85.

Frankie, G. M.; Baker, H. G. \& Opler, P. A. 1974. Comparative phenological studies of trees tropical lowland wet and dry forest sites of Costa Rica. Journal of Ecology 62: 881-931.

Gandolfi, S.; Leitão Filho, H. F. \& Bezerra, C. L. F. 1995. Levantamento florístico e caráter sucessional das espécies arbustivo-arbóreas de uma floresta mesófila semidecídua no município de Guarulhos, SP. Revista Brasileira de Biologia 55(4): 753-767.

Gibbs, P. E. \& Leitão Filho, H. F. 1978. Floristic composition of an area of gallery forest, near Mogi-Guaçu, State of São Paulo, SE Brasil. Revista Brasileira de Botânica 3: 17-22.

Grombone, M. T.; Bernacci, L. C.; Meira Neto, J. A. A.; Tamashiro, J. Y. \& Leitão Filho, H. F. 1990. Estrutura fitossociológica da floresta semidecídua de altitude do Parque Municipal de Grota Funda (Atibaia-Estado de São Paulo). Acta Botanica Brasilica 4(2): 47-64.

Jackson, J. F. 1978. Seasonality of flowering and leaf fall in a brazilian subtropical lower montane moist forest. Biotropica 10: 38-42

Jansen, D. H. 1967. Synchronization of sexual reproduction of trees within the dry season in Central America. Evolution 21: 620-637.

Köeppen, W. 1948. Climatologia. Ed. Fundo de Cultura Econômica, México - Buenos Aires.

Leitão Filho, H. F. 1982. Aspectos taxonômicos das florestas do Estado de São Paulo. Silvicultura de São Paulo 16(A): 197-206. 
Leitão Filho, H. F. 1993. Ecologia da Mata Atlântica em Cubatão, SP. Editora da Universidade Estadual Paulista (UNESP) e Editora da UNICAMP, Campinas.

Martins, F. R. 1991. Estrutura de uma floresta mesófila. Editora da UNICAMP, Campinas.

Matthes, L. A. F.; Leitão Filho, H. F. \& Martins, F. R. 1988. Bosque dos Jequitibás (Campinas, SP): composição florística e fitossociológica do estrato arbóreo. Pp. 55-76. V Congresso da Sociedade Botânica de São Paulo Anais.

Meira Neto, J. A. A.; Bernacci, L. C.; Grombone, M. T.; Tamashiro, J. Y. \& Leitão Filho, H. F. 1989. Composição florística de mata semidecídua de altitude do Parque Municipal da Grota Funda (Atibaia - Estado de São Paulo). Acta Botanica Brasilica 3(2): 51-74.

Mello, M. H. A.; Pedro Junior, M. J.; Ortolani, A. A. \& Alfonsi, R. R. 1994. Chuva e temperatura: cem anos de observações em Campinas. Boletim Técnico 154. Instituto Agronômico de Campinas.

Miranda, Z. A. I. 1996. Plano de gestão da Área de Proteção Ambiental da região de Souzas e Joaquim Egídio - APA Municipal. Relatório da Secretaria de Planejamento e Meio Ambiente de Campinas.

Morellato, L. P. C.; Rodrigues, R. R.; Leitão Filho, H. F. \& Joly, C. A. 1989. Estudo comparativo da fenologia de espécies arbóreas de floresta de altitude e floresta mesófila semidecídua na Serra do Japi, Jundiaí, São Paulo. Revista Brasileira de Botânica 12: 85-98.

Morellato, L. P. C. \& Leitão Filho, H. F. 1992. Padrões de frutificação e dispersão na serra do Japi. Pp. 112-141. In: L.P.C. Morellato (org.). História natural da Serra do Japi: Ecologia e preservação de uma área florestal no sudeste do Brasil. Editora da UNICAMP/FAPESP, Campinas.

Morellato, L. P. C. \& Leitão Filho, H. F. 1995. Ecologia e preservação de uma floresta tropical urbana. Editora da UNICAMP, Campinas.

Müller-Dumbois, D. \& Ellenberg, H. 1974. Aims and methods of vegetation ecology. John Wiley \& Sons, New York.

Oliveira-Filho, A. T.; Villela, E. A.; Carvalho, D. A. \& Gavilanes, M. L. 1994. Differentiation of streamside and upland vegetation in an area of montane semideciduous forest in southeaster Brazil. Flora 189: 287-305.

Pagano, S. N. \& Leitão Filho, H. F. 1987. Composição florística do estrato arbóreo da mata mesófila semidecídua no município de Rio Claro (Estado de São Paulo). Revista Brasileira de Botânica 10: $37-47$.
Penhalber, E. F. \& Mantovani, W. 1997. Floração e chuva de sementes em mata secundária em São Paulo, SP. Revista Brasileira de Botânica 20(2): 205-230.

Pielou, E. C. 1984. The interpretation of ecological data. A primer on classification and ordination. Wiley, New York.

Ramirez, N. 1993. Producion y costo de frutos y semillas entre formas de vida. Biotropica 25(1): 46-60.

Rodrigues, R. R.; Morellato, L. P. C.; Joly, C. A. \& Leitão Filho, H. F. 1989. Estudo florístico e fitossociológico em um gradiente altitudinal de mata estacional mesófila semidecídua, na Serra do Japi, Jundiaí, SP. Revista Brasileira de Botânica 12: 71-84.

Rossi, L. 1994. A flora arbórea-arbustiva da mata da reserva da Cidade Universitária "Armando de Salles Oliveira” (São Paulo, Brasil). Boletim do Instituto de Botânica n. 9. Instituto de Botânica, São Paulo.

Rozza, A. F. 1997. Florística, fitossociologia e caracterização sucessional em uma floresta estacional semidecidual: Mata da Virgínia, Matão, SP. Dissertação de Mestrado. Universidade Estadual de Campinas, Campinas.

Salis, S. M.; Tamashiro, G. J. Y. \& Joly, C. A. 1994. Florística e fitossociologia do estrato arbóreo de um remanescente de mata ciliar no Rio Jacaré Pepira, Brotas, SP. Revista Brasileira de Botânica 17(2): 93-103.

Salis, S. M.; Shepherd, G. J. \& Joly, C. A. 1995. Floristic comparison of mesophytic semideciduous forests of the interior of the State of São Paulo, Southeast Brazil. Vegetatio 119: 155-164.

Santin, D. A. 1999. A vegetação remanescente do município de Campinas (SP): mapeamento, caracterização fisionômica e florística, visando a conservação. Tese de Doutorado. Universidade Estadual de Campinas, Campinas.

Shepherd, G. J. 1995. FITOPAC 1. Manual do usuário. Departamento de Botânica. Instituto de Biologia, Universidade Estadual de Campinas, Campinas.

Silva, A. F. 1989. Composição florística e estrutura fitossociológica do estrato arbóreo da Reserva Florestal Augusto Ruschi, São José dos Campos, SP. Tese de Doutorado. Universidade Estadual de Campinas, Campinas.

Silva, A. F. \& Leitão Filho, H. F. 1982. Composição florística e estrutura de um trecho da mata atlântica de encosta no município de Ubatuba (São PauloSP). Revista Brasileira de Botânica 5: 43-52. 
Spina, A. P. 1997. Composição florística da mata higrófila na região de Campinas. Dissertação de Mestrado. Universidade Estadual de Campinas, Campinas.

Stranghetti, V. 1996. Levantamento florístico das espécies vasculares de uma floresta estacional no norte do Estado de São Paulo, Estação Ecológica de Paulo de Faria. Tese de Doutorado. Universidade Estadual de Campinas, Campinas.

Tamashiro J. Y.; Rodrigues, R. R. \& Shepherd, G. J. 1986. Estudo florístico e fitossociológico da Reserva da Mata de Santa Genebra, Campinas SP. Relatório de Pesquisa. Fundação de Amparo à Pesquisa de São Paulo.
Toniato, M. T.; Leitão Filho, H. F. \& Rodrigues, R. R. 1998. Fitossociologia de um remanescente de floresta higrófila (mata de brejo) em Campinas. Revista Brasileira de Botânica 21(2): 197-210.

Torres, R.B. 1989. Estudos florísticos em mata secundária do sítio Ecológica de Angatuba; Angatuba (São Paulo). Dissertação de Mestrado. Universidade Estadual de Campinas, Campinas.

Torres, R. B.; Matthes, L. A. F. \& Rodrigues, R. R. 1994. Florística e estrutura do componente arbóreo de mata de brejo de Campinas, SP. Revista Brasileira de Botânica 17(2): 189-194.

Torres, R. B.; Martins, F. R. \& Kinoshita, L. S. 1997. Climate, soil and tree flora relationships in forests in the State of São Paulo, southeastern Brasil. Revista Brasileira de Botânica 20(1): 41-51. 